Acta Protozool. (2017) 56: 129-137

\title{
Rat spleen in the course of Babesia microti invasion: histological and submicroscopic studies
}

\author{
Hubert OKŁA ${ }^{1}$, Krzysztof P. JASIK ${ }^{2}$, Danuta URBAŃSKA-JASIK ${ }^{3}$, Jan SŁODKI ${ }^{2}$, Beata \\ ROZWADOWSKA ${ }^{2,4}$, Michal GRELOWSKI ${ }^{5,6}$, Ewa CHMIELIK ${ }^{7}$, Aleksandra SŁODKI ${ }^{2}$, Marta \\ ALBERTYŃSKA ${ }^{2,4}$, Aniela GRAJOSZEK ${ }^{8}$
}

${ }^{1}$ Institute of Materials Science, Faculty of Computer Science and Materials Science, University of Silesia in Katowice, Poland;
${ }^{2}$ Department of Skin Structural Studies, School of Pharmacy with the Division of Laboratory Medicine in Sosnowiec, Medical
University of Silesia in Katowice, Poland; ${ }^{3}$ Department of Animal Histology and Embryology, Faculty of Biology and Environmental
Protection, University of Silesia in Katowice, Poland; ${ }^{4}$ Provincial Sanitary and Epidemiological Station in Katowice, Poland;
${ }^{5}$ August Chelkowski Institute of Physics, Faculty of Mathematics, Physics and Chemistry, University of Silesia in Katowice, Poland;
${ }^{6}$ Department of Biophysics and Morphogenesis of Plants, Faculty of Biology and Environmental Protection, University of Silesia in
Katowice, Poland; ${ }^{7}$ Tumor Pathology Department, Maria Sklodowska-Curie Memorial Cancer Center and Institute of Oncology,
Gliwice Branch, Poland; ${ }^{8}$ Department for Experimental Medicine, School of Medicine in Katowice, Medical University of Silesia
in Katowice, Poland

Abstract. The course of babesiosis in humans is characterized by various intensity levels - from a subclinical level to the severe one - associated with multiple organ failure, which leads to death. The aim of this study was to evaluate the effect of 21-day and 6-month invasion of B. microti on Wistar rats spleen. Histological changes in the rats' spleen were characterized by swelling of splenic tissue, especially the tissue adjacent to the capsule. In the structure of the white pulp in some rats, high concentrations of lymphocytes occurred. The boundary between the white pulp and red pulp was blurred. In the red pulp structure of rats, a lot of macrophages and extracellular deposits of bilirubin were present. The submicroscopic studies showed that the nuclear matrix was slightly shrunken. In the red pulp fragments of the damaged cells were located in the intercellular spaces. Near these areas, many thrombocytes were visible. The ultrastructural observation also revealed thickened endoplasmic reticulum membranes, local cellular swelling filled with amorphous substance, and digested erythrocytes. B. microti invasion affects the splenic morphology and ultrastructure in rats. The immunological hyperactivity and signs of inflammation indicate an important role of spleen in a fight against parasites.

Key words: Babesia microti, rat spleen, histology, ultrastructure, atomic force microscopy

Addresses for correspondence: Hubert Okła - Institute of Materials Science, Faculty of Computer Science and Materials Science, University of Silesia in Katowice, 75 Pułku Piechoty 1A, 41-500 Chorzów, Poland. Tel.: +48 32 3497522; fax: +48 323497594. E-mail address: hubert.okla@gmail.com.
Krzysztof Jasik - Department of Skin Structural Studies, School of Pharmacy with the Division of Laboratory Medicine in Sosnowiec, Medical University of Silesia in Katowice, Kasztanowa 3, 41-200 Sosnowiec, Poland. Tel.: +48 32 2699834; fax: +48 322699834 . E-mail:kjasik@sum.edu.pl. 


\section{INTRODUCTION}

Babesiosis, apart from Lyme disease (called borreliosis) and tick-borne encephalitis, is one of the most important zoonoses transmitted by ticks. The etiologic factors of the disease are protozoans belonging to the genus Babesia (phylum: Apicomplexa, order: Piroplasmida, family: Babesiidae), which enter the blood via the tick saliva, and invade erythrocytes (Sonenshine 1993). It is estimated that in some regions, the morbidity rate of babesiosis is equal with the morbidity rate of borreliosis (Gajda et al. 2015). Most of the cases of human babesiosis in the world are caused by $B$. microti (Teal et al. 2011). The Centers for Disease Control and Prevention (CDC) added babesiosis to notifiable diseases due to the constantly increasing number of cases in North America (Lobo et al. 2013).

The course of babesiosis in humans is characterized by various intensity levels - from a subclinical level to the severe one - associated with multiple organ failure, which leads to death (Zaidi and Singer 2002). Typically, the protozoan invasion causes a disease with mild flu-like symptoms similar to malaria (Kuzna-Grygiel and Kolodziejczyk 2000). A mild, often asymptomatic course is observed in immunocompetent patients, whereas in immunocompromised patients, a severe disease may appear (Kuzna-Grygiel and Kolodziejczyk 2000, Vannier et al. 2008, Torres-Velez et al. 2003, Gajda et al. 2015).

The severe course of babesiosis usually results in serious complications in many organs, which cause death in the final stage. The most common complications are liver and kidney damage, respiratory failure, congestive heart failure, myocardial infarction, disseminated intravascular coagulation syndrome, spontaneous atraumatic splenic rupture or splenic infarction (Kuwayama and Briones 2008, Vannier et al. 2008, Gajda et al. 2015, Mylonakis 2001). The damage of spleen may not necessarily be associated with splenomegaly (Kuwayama and Briones 2008).

In the spleen, the mechanisms of nonspecific and specific immune response against protozoa occur, therefore, the splenectomy is associated with the acute course of babesiosis. Furthermore, invaded erythrocytes and metabolites of Babesia spp. are eliminated in this organ (Adaszek et al. 2014). When parasitaemia is up to $20 \%$, jaundice often develops as a result of intense erythrocyte hemolysis (Homer et al. 2000, Gajda et al. 2015). The pulmonary oedema and acute respira- tory distress syndrome are also observed in severe invasion (Homer et al. 2000, Gajda et al. 2015, Mørch et al. 2015). Less common complications include dysfunction of the central nervous system, such as emotional lability or depression (Vannier et al. 2008, Kuzna-Grygiel and Kolodziejczyk 2000). The risk of the complicated course of babesiosis grows with increasing level of parasitemia, however the exact pathophysiological mechanism of complications in babesiosis has not been explained yet (Gajda et al. 2015).

The aim of this study was to evaluate the effect of 21-day and 6-month (transient and long-lasting, respectively) invasion of $B$. microti on Wistar rats' spleen. The experiment included histological and submicroscopic analyses of the splenic tissue.

\section{MATERIALS AND METHODS}

\section{Experimental design}

In the presented study, 20 Wistar rats invaded with a reference strain of B. microti (ATCC 30221) were used. The inoculation procedure involved injecting intraperitoneally $0.5 \mathrm{ml}$ blood obtained from rats with confirmed parasitemia. The moment of injection was regarded as a beginning of the invasion.

The invaded rats were divided into two groups, each of which consisted of 10 individuals. The first group consisted of rats in which invasion lasted 21 days, while it was 6 months in the second group. Two healthy rats were a control group. During experimental procedure, $0.5 \mathrm{ml}$ of sodium chloride physiological solution for intravenous injection (Inj. Natrii Chlorati Isotonica Polpharma, $9 \mathrm{mg}$ $\mathrm{ml}$, Polpharma, Starogard Gdański, Poland) was injected intraperitoneally into the control rats. The invaded and control rats were housed in standardized zoohygienic conditions (Okła et al. 2014). In vivo studies were conducted in accordance with resolution No. 32/2011 of the Local Ethics Committee for Animal Experiments in Katowice, Poland. All procedures were carried out according to the protocol based on the European Convention for the Protection of Vertebrate Animals used for Experimental and Other Scientific Purposes.

\section{Parasitemia monitoring}

The parasitemia monitoring was performed in both study groups at different intervals using microscopic analyses of blood smears. Blood smears were fixed in methyl alcohol (POCh, Gliwice, Poland) and stained with Giemsa and May-Grünwald solutions (Aqua-Med ZPAM, Cracow, Poland) according to the manufacturer's instructions. The dried preparations were observed using Olympus BX60 microscope. The assessment of parasitemia was conducted according to recommendations attached to the specification of the reference strain of $B$. microti. This procedure was based on a microscopic observation of 500 consecutively encountered 
erythrocytes. The cytoplasm of invaded erythrocytes contained a basophilic inclusions responding to Babesia spp. merozoites.

Atomic force microscopy (AFM) was performed in order to measure and analyze the surface of peripheral blood smear of the rats. The unfixed, dried rat blood smears collected immediately prior to animal euthanasia were used in topographical studies. AFM measurement was performed using NanoWizard ${ }^{\circledR} 3$ BioScience atomic force microscope (JPK Instruments AG, Berlin, Germany) combined with inverted optical microscope, i.e. Axio Observer (Zeiss, Jena, Germany). The AFM was located in a specially designed laboratory with acoustic and vibration isolation to ensure absolute stability. The optical microscope with differential interference contrast was used in order to position the AFM tip to the desired location on the smear surface. All experiments were performed in air using the intermittent contact mode ( $\mathrm{AC}$ mode) with the $\mathrm{Z}$ range set to $15-\mu \mathrm{m}$ and a scan rate of $0.5-1 \mathrm{~Hz}$. Imaging was carried out using the Tapping Mode NSC15/AL BS etched silicon probes with a resonance frequency 265-410 kHz (MikroMasch, Portland, USA). The drive amplitude and set point were adjusted during the measurement to minimize tip artefacts. The JPK SPM Software (JPK Instruments AG, Berlin, Germany) was used for AFM operation and imaging process.

\section{Structural and ultrastructural analyses}

21 days and 6 months after inoculation of protozoan or saline injection, the blood was collected from the caudal vein and stored at $4{ }^{\circ} \mathrm{C}$. Then the rats were euthanized using isoflurane for inhalational anesthesia (Forane ${ }^{\circledR}$-Baxter, Deerfield, IL, USA). During post-mortem examination, the samples of spleen were collected and fixed in appropriate solutions.

The spleen samples for structural analysis were fixed in Bouin's solution. The sections stained by the Masson trichrome method were analyzed using an Olympus BX60 microscope equipped with XC50 digital camera and Olympus cellSens Standard software.

The material for ultrastructural studies was fixed in Karnovski's solution. The submicroscopic observations were performed using a transmission electron microscope Hitachi H500 at $75 \mathrm{kV}$. The standard methodology of the tissue preparation for histological and ultrastructural studies was used (Okła et al. 2014).

The first stage involved the separation of blood cells by centrifugation at low speed. Than the cells were fixed in $2.5 \%$ paraformaldehyde solution at $4{ }^{\circ} \mathrm{C}$. Further procedures for ultrastructural analysis followed standard methodology. The blood cells samples for transmission electron microscopy were prepared in the same way as samples of the spleen.

\section{RESULTS}

The microscopic observation of peripheral blood smears stained by May-Grünwald-Giemsa (MGG) method demonstrated signs of invasion in rats from both study groups (transient and long-lasting invasion). The intraerythrocytic, basophilic, irregular inclusions were observed in many fields of analysis (Figs 1A-C).

The measurement of blood smears topography collected from rats with transient and long-lasting inva- sion of B. microti using AFM allowed to explore the cells surface and provided information about their size and shape. Blood cells imaging from both study groups demonstrated invaded erythrocytes which were characterized by irregular surface. More detailed analysis allowed to observe the characteristic, elongated structures (about $2 \mu \mathrm{m}$ in length) under the cell membrane. The shape of these structures resembled $B$. microti merozoites. Thanks to the use of the AFM, it was possible to eliminate the influence of the fixer and obtain high resolution (Figs 1D, E).

\section{Microscopic changes in rat spleen with $B$. microti invasion}

The capsule of the rat spleen with 21-day B. microti invasion was characterized by the presence of a small amount of connective tissue, coated with a layer of mesothelial cells. Most rodents from the study group had degenerative changes characterized by swelling (oedema, hydropic changes, vacuolar degeneration). It was observed especially in the structure of the splenic tissue adjacent to the capsule.

Moreover, microscopic observations demonstrated that the capsule was detached from the rest of the organ. The splenic trabeculae emerged from the capsule, and they were barely visible in the deeper layers of the spleen (Fig. 2A).

A different situation was observed in the structure of the rat spleen with 6-month B. microti invasion. In this case, the capsule was thicker than in organs of rodents with transient parasitemia. Numerous thick connective fibers were found in the structure. In the splenic tissue, a lot of splenic trabeculae emerged from the capsule and branched out. (Fig. 2B). In addition, hyperplasia of mesothelial cells was observed in the spleen. A lot of single connective fibers were visible in deeper layers. The fibers penetrated splenic cells more deeply than in control organs (Figs 2C, D).

In the structure of the white pulp of rats with transient parasitemia, higher concentrations of lymphocytes than in the control group occurred. The shape of lymphoid follicles was irregular. In the marginal regions of the spleen, follicles bored towards the capsule. In addition, they had wide germinal centers and marginal zones. The boundary between the white pulp and red pulp, seemed to be blurred in some rodents. The wall of central arteries was often thickened and showed characteristics of reactivity (Fig. 2E). In this part of the spleen, swelling also occurred. 


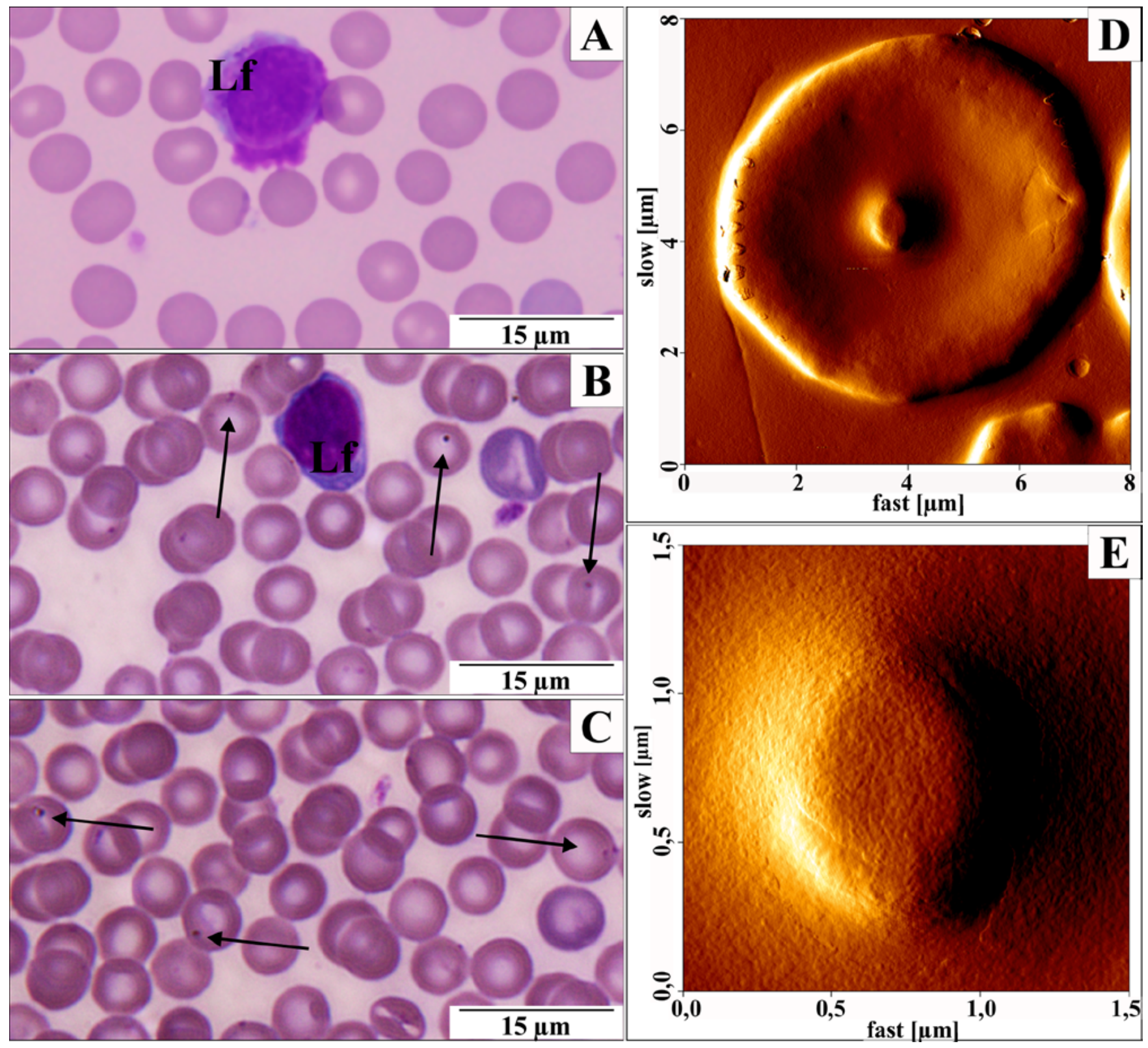

Figs 1A-E. The peripheral blood smear of control rats (A) rats with 21-day B. microti invasion (B) and rats with 6-month B. microti invasion (C) (black arrows $-B$. microti merozoites). Preparations were stained with MGG method. The surface observations of erythrocytes invaded with $B$. microti showed the presence of characteristic, elongated structures under the cell membrane (D, E). Imaging in AFM. Abbreviation: Lf - lymphocyte.

Figs 2A-F. A subcapsular part of rat spleen with 21-day (A, arrows show stratification under the splenic capsule) and 6-month $B$. microti invasion (B). The splenic pulp of control rats $(\mathbf{C})$ and rat spleen with 6-month $B$. microti invasion (D) - connective fibers are visible (arrows). Lymphoid follicles in the spleen of rats with 21-day (E) and 6-month B. microti invasion (F). Preparations were stained with the Masson trichrome method. Abbreviations: Caps - spleen capsule composed of connective tissue and smooth muscle, CAr - central artery, Nod - splenic nodule. 
Rat spleen in the course of Babesia microti invasion $\mathbf{1 3 3}$

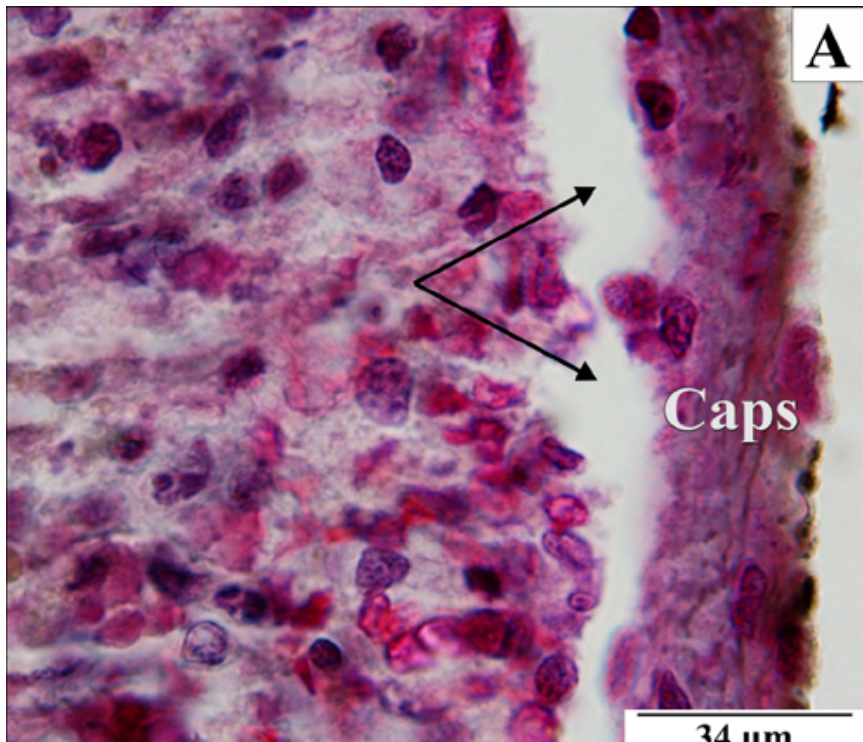

F)

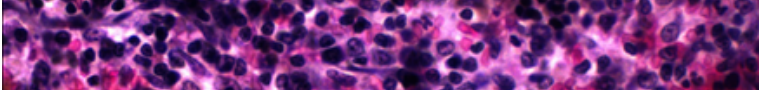
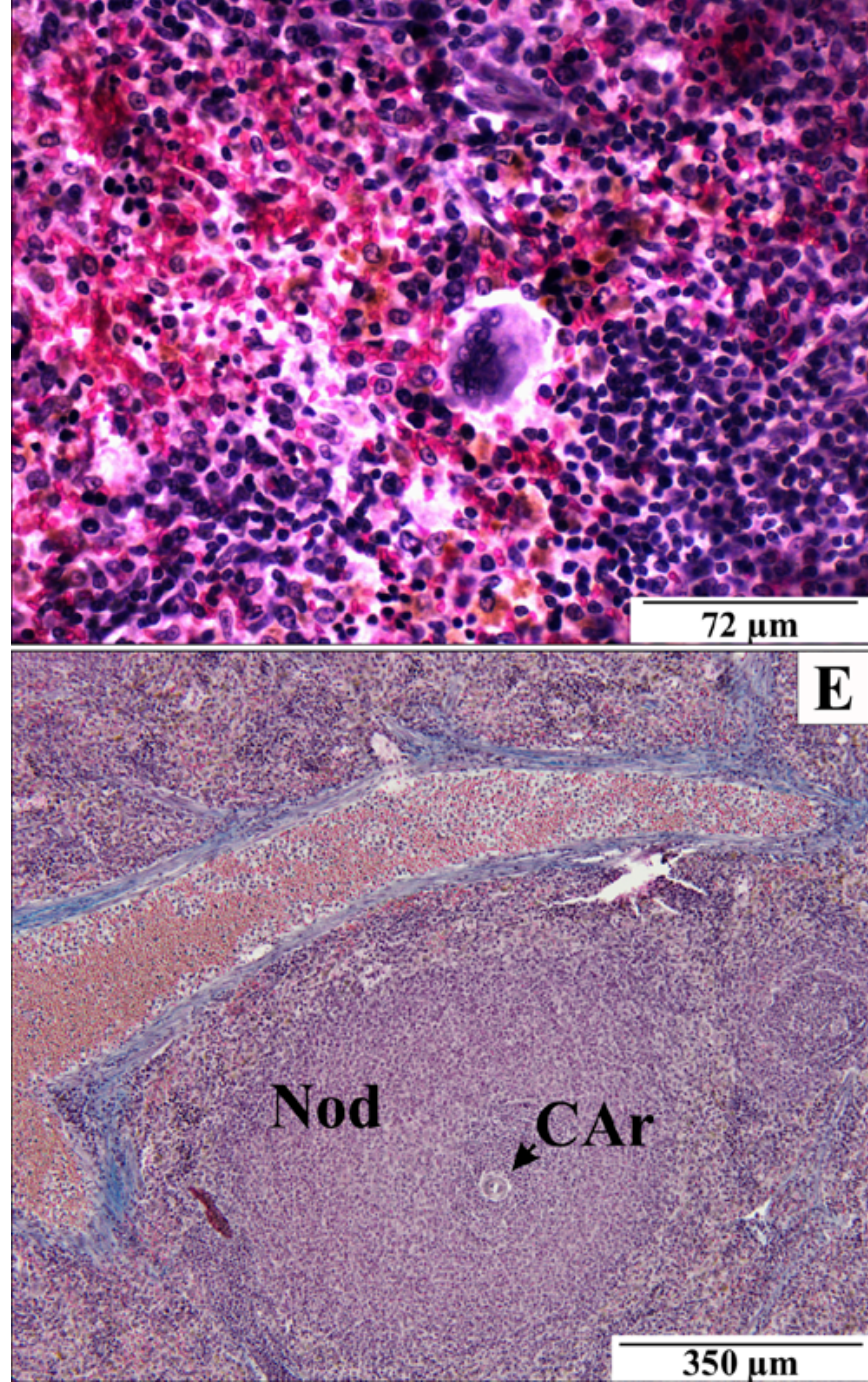

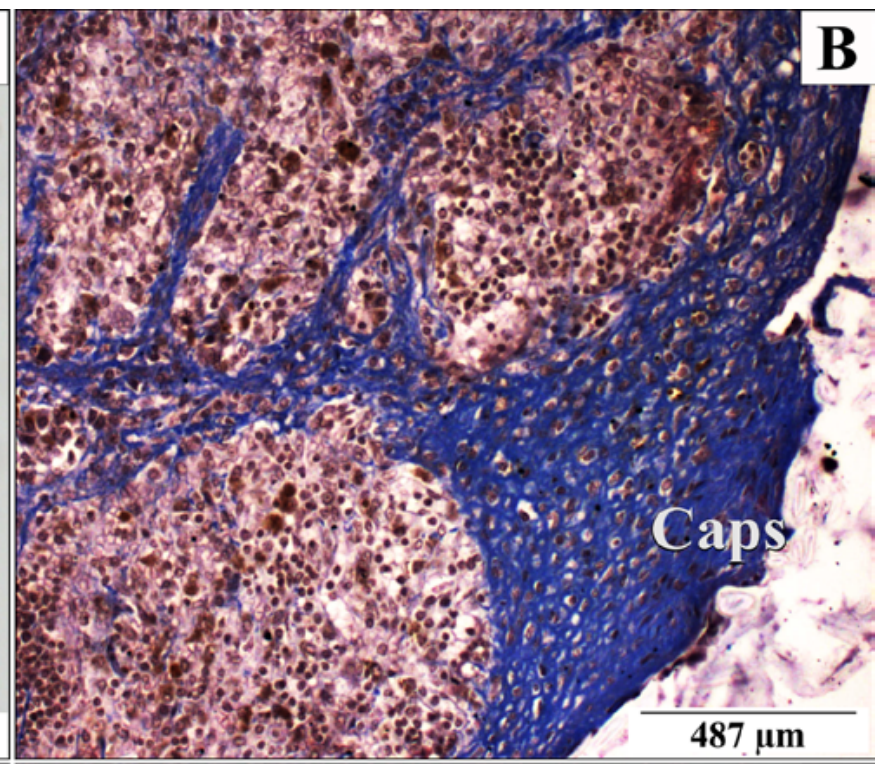

62 .

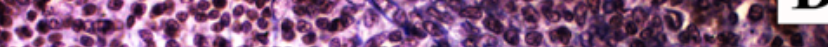

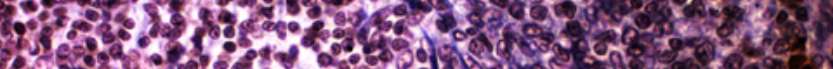

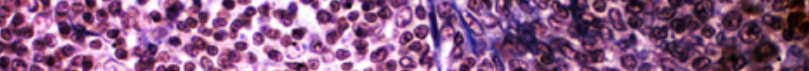
70 :

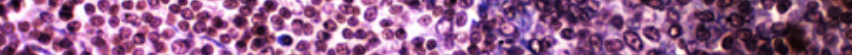

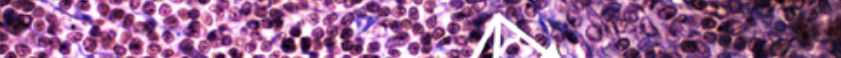

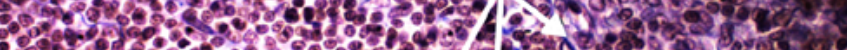

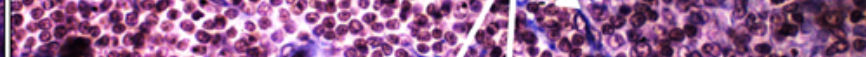

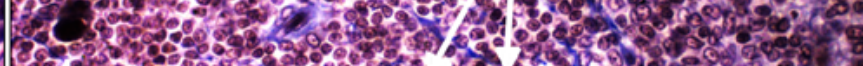

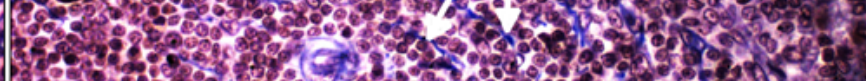
Et

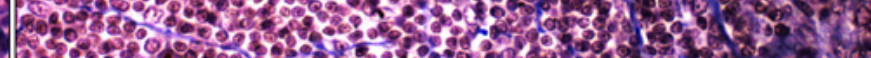
2.

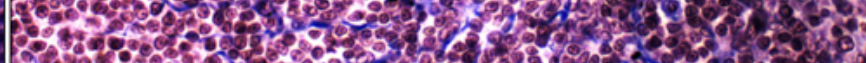

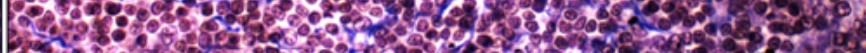

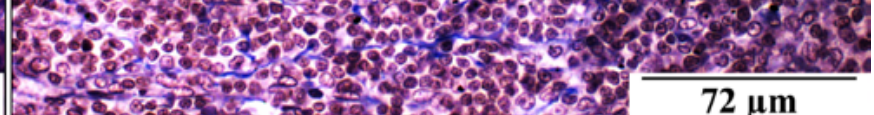

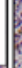

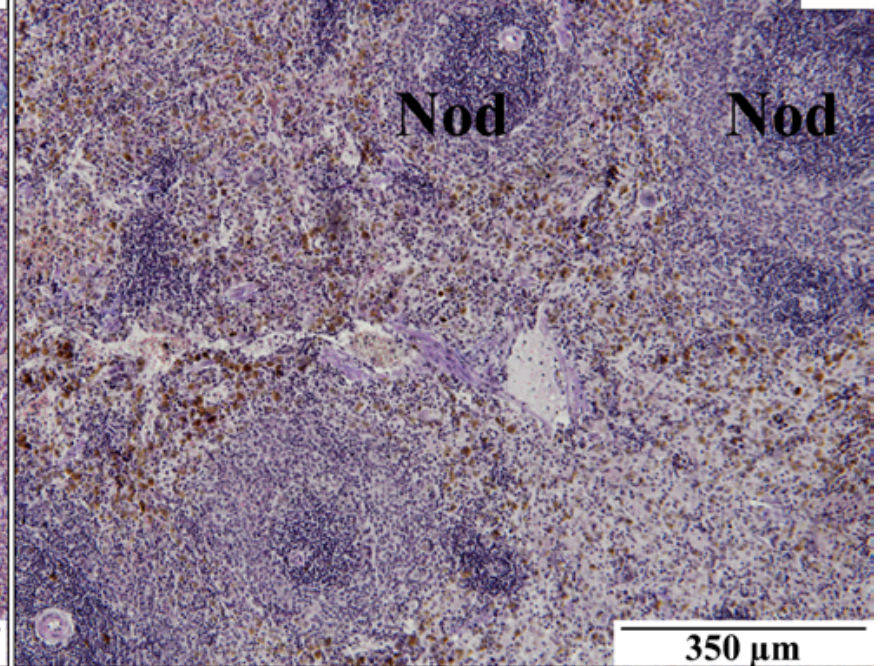


In the case of the rat spleen with long-lasting parasitemia, fewer follicles were visible in the white pulp compared to organs collected from rats with transient B. microti invasion. The follicles were smaller and had tighter marginal zones, which in some areas were completely blurred and blended with the red pulp. The wall of blood vessels located in the white pulp was also thickened, which could be related to the increase in the total amount of connective tissue in the spleen (Fig. 2F).

By contrast, in the red pulp structure of rats with 21-day B. microti invasion, many erythrocytes with different staining of cytoplasm occurred. A lot of macrophages and extracellular deposits of bilirubin were observed nearby. Some macrophages contained cytoplasmic inclusions of hemosiderin.

The same changes were observed in the course of the 6-month invasion of B. microti, however they were characterized by greater intensity. The red pulp constituted a greater part of the splenic tissue. A tendency to the accumulation of bilirubin, especially around the border between red and white pulp, was also observed. Furthermore, swellings were visible in many areas where the fluid had been accumulated. However, these changes were not as intensive as in the case of the rat spleen with transient parasitemia. It is worth noting that no rat spleen was enlarged.

\section{Ultrastructural changes in the rat spleen with $B$. mi- croti invasion}

The submicroscopic studies of the rat spleen in both study groups (the transient and long-lasting invasion of $B$. microti) confirmed loosening of the organ structures. In many areas, microcracks were visible. The empty spaces in the nuclear envelope occurred in splenic tissue obtained from rats with 21-day invasion of $B$. microti. The nuclear matrix seemed to be slightly shrunken. The changes were observed especially in the white pulp. The ultrastructural studies confirmed swelling. In many areas of observation, empty spaces were filled with cellular debris. An analogous ultrastructural changes were visible in the rat spleen with long-lasting invasion. However, in some organs, the nuclei were intensely shrunken, and the continuity of the nuclear membrane were disintegrated. The nuclei had pyknotic shape (Fig. 3A).

The submicroscopic structure of red pulp in rat spleen with transient parasitemia demonstrated pathological changes mainly around the blood vessels. Fragments of the damaged cells were located in the intercellular spaces (Fig. 3B). Near these spaces, many thrombocytes were visible (Fig. 3C). In many areas of observation, disintegrated erythrocytes could be seen. Structural elements of $B$. microti merozoites constituted the characteristic inclusions in the cytoplasm (Fig. 3D). Many degenerated splenic cells were also visible in the area of blood vessels, whose cytoplasm contained a lot of glycogen, residual bodies and multi-vesicular bodies containing electron-dense structures. In addition, the ultrastructural observation demonstrated thickened endoplasmic reticulum membranes, local cellular swelling filled with amorphous substance, and digested erythrocytes. In some vacuoles, fragments of cytoskeleton and remains of cell membranes were detected. In the course of long-lasting parasitemia in the ultrastructure of the rat spleen, especially in the red pulp, swellings were also observed, among them: vacuoles filled with amorphous substance and residual bodies and multivesicular bodies. In many areas, the mitochondria were damaged. Near the vacuoles, platelets which adhered to the surface of a damaged cells, were visible (Fig. 3E).

In other areas of observation, many macrophages were seen. In the cytoplasm of these cells, multiple autofagosomes containing shrunken erythrocytes occurred. In the surrounding regions, strong osmophilic granules, which included intracellular deposits of hemosiderin, were located (Fig. 3F). Similar changes occurred in both study groups.

\section{DISCUSSION}

In Europe, babesiosis is mainly perceived as an animal disease, but recently the awareness that Babesia spp. parasites can cause diseases in humans has increased. Ticks, the main vectors of parasites, live in areas of human life activity, not only in forests but also in urban parks and recreational areas. The resent studies have demonstrated an increasing number of ticks which transmit Babesia spp. Therefore, babesiosis has been included in the group of emerging invasive diseases (Gray et al. 2002, Homer et al. 2003, Skotarczak 2007, Leiby 2011). From an epidemiological point of view, two species: $B$. divergens and $B$. microti are the most important for humans (Karbowiak 2004). B. microti is an etiological agent of the largest number of cases of human babesiosis in the world (Teal et al. 2011).

The microscopic and ultrastructural observations of rat erythrocytes demonstrated cell membrane damage in the course of parasitemia. Herwaldt et al. (2011) 

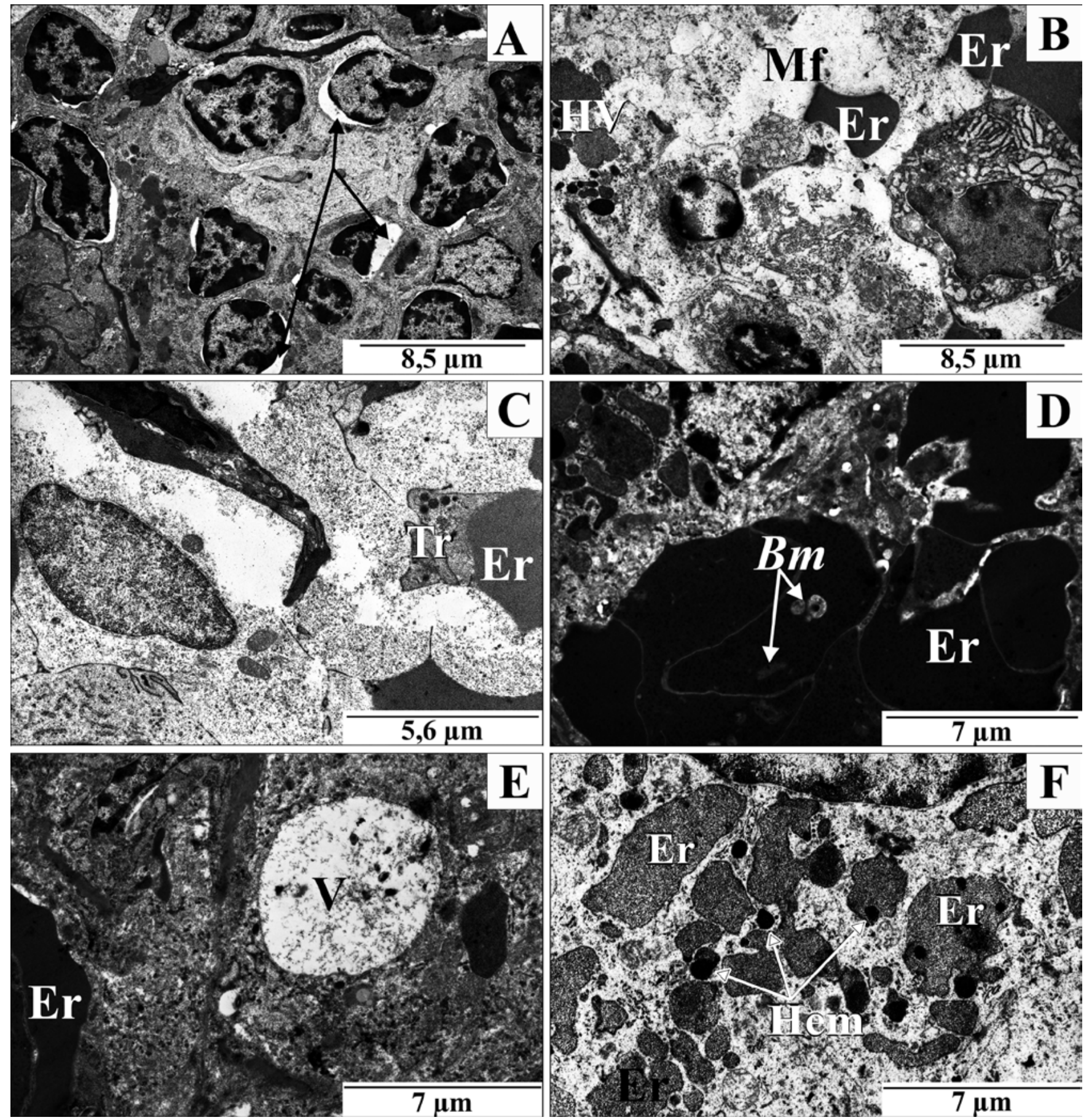

Figs 3A-F. Splenic white pulp of rats with 21-day (A, arrows show empty spaces in nuclear membrane) and 6-month $B$. microti invasion (B). Swellings in rat spleen with 21-day B. microti invasion (C). Invaded erythrocytes in sinus blood vessels in rat spleen with 21-day parasitemia (D). Vacuole in macrophage of the rat spleen with 6-month $B$. microti invasion $(\mathbf{E})$. Macrophage in red pulp of the rat spleen with 6-month $B$. microti invasion (F). Preparations imaging with the use of transmission electron microscopy (TEM). Abbreviations: $B m$ - Babesia microti merozoites, Er - erythrocytes, Hem - hemosiderin, Mf - macrophage containing digested fragments of erythrocytes and heterophagical vacuoles $-\mathrm{HV}, \mathrm{Tr}$ - thrombocytes, $\mathrm{V}$ - vacuole containing fibrous remnants of cytoskeleton. 
indicated that free radicals synthesized during intracellular divisions of Babesia spp. merozoites may be the cause of such damage. In further stages of babesiosis, hemolytic anemia usually occurs, and causes hyperbilirubinuria and hemoglobinuria (Homer et al. 2000, Lodes et al. 2000, Gray et al. 2002, Hunfeld 2008, Gray et al. 2010, Lobo et al. 2012, Mosqueda et al. 2012). The use of transmission electron microscopy for ultrastructural analyses of cellular blood components in rats invaded with $B$. microti showed a number of erythrocytes with ring structures inside the cytoplasm. Similar electrongrams were presented by Montero et al. (2009) who, for the first time, investigated the ultrastructural studies of human erythrocytes invaded with B. divergens.

The cell divisions in the cytoplasm by schizogony give a number of daughter merozoites. The topography measurement of peripheral blood smear surface confirmed the presence of sharp, pear-shaped forms under the erythrocyte cell membrane. The knobby structures on the surface of invaded cells participate in erythrocytes agglomeration, and adhesion to endothelial cells. The erythrocytes sequestration leads to the closure of small vessels resulting in rheological, organ and metabolic consequences (Knap and Myjak 2008).

From the immunological point of view, spleen seems to be the most important organ. Kuwayama and Briones (2008) described a case of a 61-year-old man with nonspecific symptoms. Laboratory examination detected Babesia merozoites in his blood. The spleen was very soft and extremely fragile. A histological analysis of the removed organ demonstrated changes mainly in the red pulp. The authors marked an increase in the number of plasma cells, lymphocytes, immunoblasts and histiocytes which demonstrated signs of hyperplasia. It is worth noting that the organ was not enlarged. Similar results were obtained by Schneider et al. (2011) who conducted a study of $B$. bovis in an animal model. However, the spleen of calves with acute invasion of $B$. bovis was enlarged, and hyperplastic nucleated cells were visible in the splenic structure.

Our results presented in this study confirmed the increased activity of rat spleen in the course of invasion, mainly during the first stage. In both study groups, the structure of organs had noticeable signs of an intense immune response. When both groups were compared, phases of morphological changes in the course of $B$. microti invasion were observed. In the case of transient parasitemia, the number of lymphocyte groups and macrophages increased in the spleen. In some rats, many swellings, often filled with fluid, were visible. With the extension of parasitemia duration, a number of these changes decreased, however the amount of connective tissue increased. This situation resulted in fibrosis of the spleen. In some spleen structures, signs of cirrhosis occurred, and they were associated with excessive fibrosis and loss of the white pulp at the expense of the red pulp. These symptoms may indicate a slow atrophy process of the spleen in the course of $B$. microti invasion. A large amount of fluid secreted into the intercellular spaces, mainly in rats with 21-day invasion, can cause a pressure increase inside the spleen.

The observations published by Dkhil et al. (2014) confirmed an important role of the spleen in the course of $B$. divergens in Mongolian gerbils (Meriones unguiculatus). Collected organs had signs of hyperreactivity resulting in enlargement. Histological analyses demonstrated that the white pulp underwent expansion, the border with the red pulp was blurred and cells located nearby were swollen. The hemosiderin deposits were also observed. A second aspect of the parasitemia in gerbils, described by Dkhil et al. (2014), was the role of reactive oxygen species in tissue damage. In addition, the authors confirmed that $B$. divergens invasion affected the cell cycle. In the spleen, the amount of cells in the $\mathrm{S}$ and $\mathrm{G} 2 / \mathrm{M}$ phase were increased compared to healthy animals.

The degradation of intracellular protozoan forms is probably caused by T-lymphocytes, which secrete interferon gamma (IFN- $\gamma$ ), especially the population of cells expressing the CD4 glycoprotein on the surface. The mechanism of parasitemia inhibition by IFN- $\gamma$ is not fully known. Among other mechanisms of defense against invasion, we can mention innate immune response, especially macrophages and NK cells which are able to produce soluble mediators in response to different, non-specific infectious agents. NK cells are mostly responsible for the fight against disease in the early stages of parasitemia (Homer et al. 2000). The case presented by Shaio et al. (1998) confirmed that an increased activity of NK cells was observed in patients with acute babesiosis.

Macrophages also play an important role in the fight against Babesia spp. The study published by Li et al. (2012), which was conducted on mice with INF- $\gamma$ deficiency combined with severe immunodeficiency caused by $B$. microti and B. rodhaini invasion, confirmed that the inactivation of macrophages resulted in parasitemia, and an increase in the rodent mortality rates. Similar results were described by Terkawi et al. 
(2015), who also checked the influence of macrophages depletion in the course of the invasion during different periods of parasitemia. The treatment with clodronate liposome was conducted in order to induce apoptosis of macrophages. The macrophages depletion in the initial, acute phase of the invasion resulted in a significant increase of parasitemia, which was associated with high mortality in experimental animals. However, the macrophages depletion in the later phase of the disease also caused more acute symptoms. It was associated with an increased degree of parasitemia. In the case of a latent phase, manifestation of symptoms which caused a slight increase in mortality of mice was observed.

In conclusion, $B$. microt $i$ invasion affects the splenic morphology and ultrastructure in rats. Changes intensify with the parasitemia prolongation. The immunological hyperactivity and signs of inflammation, especially in transient invasion of $B$. microti, indicate an important role of the spleen in fight against parasites. The changes in rat spleen with long-lasting parasitemia confirmed the reduction of immune function.

\section{REFERENCES}

Adaszek Ł., Łojszczyk-Szczepaniak A., Dzięgiel B., Bartnicki M., Kalinowski M., Winiarczyk S. (2014) Rola śledziony w babeszjozie. Życie Weterynaryjne. 89: 671-674 (in Polish)

Dkhil M. A., Al-Quraishy S., Al-Khalifa M. S. (2014) The effect of Babesia divergens infection on the spleen of Mongolian gerbils. Biomed. Res. Int. 2014: 1-7

Gajda P., Rustecka A., Kalicki B. (2015) Human babesiosis - a little-known tick-borne disease. Pediatr. Med. Rodz. 11: 75-82 (in Polish)

Gray J., von Stedingk L. V., Granström M. (2002) Zoonotic babesiosis. Int. J. Med. Microbiol. 291: 108-111

Gray J., Zintl A., Hildebrandt A., Hunfeld K. P., Weiss L. (2010) Zoonotic babesiosis: Overview of the disease and novel aspects of pathogen identity. Ticks Tick Borne Dis. 1: 3-10

Herwaldt B. L., Linden J. V., Bosserman E., Young C., Olkowska D., Wilson M. (2011) Transfusion-associated babesiosis in the United States: a description of cases. Ann. Intern. Med. 155: 509-519

Homer M. J., Aguilar-Delfin I., Telford S. R. 3rd, Krause P. J., Persing D. H. (2000) Babesiosis. Clin. Microbiol. Rev. 13: 451-469

Homer M. J., Lodes M. J., Reynolds L. D., Zhang Y., Douglass J. F., McNeill P. D., Houghton R. L., Persing D. H. (2003) Identification and characterization of putative secreted antigens from Babesia microti. J. Clin. Microbiol. 41: 723-729

Hunfeld K. P., Hildebrandt A., Gray J. S. (2008) Babesiosis: recent insights into an ancient disease. Int. J. Parasitol. 38: 1219-1237

Karbowiak G. (2004) Zoonotic reservoir of Babesia microti in Poland. Pol. J. Microbiol. 53(Suppl.): 61-65

Knap J. P., Myjak P. (Eds.) (2008) Malaria. Profilaktyka, klinika, zwalczanie. Główny Inspektorat Sanitarny, Warszawa (in Polish)

Kuwayama D. P., Briones R. J. (2008) Spontaneous splenic rupture caused by Babesia microti infection. Clin. Infect. Dis. 46: e92-95
Kuzna-Grygiel W., Kolodziejczyk L. (2000) Studies of liver of mice in the course of an experimental infection with Babesia microti. I: Histopathological changes and changes in the glycogen content. J. Protozool. Res. 10: 14-23

Leiby D. A. (2011) Transfusion-transmitted Babesia spp.: Bull'seye on Babesia microti. Clin. Microbiol. Rev. 24: 14-28

Li Y., Terkawi M. A., Nishikawa Y., Aboge G. O., Luo Y., Ooka H., Goo Y. K., Yu L., Cao S., Sun Y., Yamagishi J., Masatani T., Yokoyama N., Igarashi I., Xuan X. (2012) Macrophages are critical for cross-protective immunity conferred by Babesia microti against Babesia rodhaini infection in mice. Infect. Immun. 80: $311-320$

Lobo C. A., Cursino-Santos J. R., Alhassan A., Rodrigues M. (2013) Babesia: An emerging infectious threat in transfusion medicine. PLoS Pathog. 9: e1003387

Lobo C. A., Rodriguez M., Cursino-Santos J. R. (2012) Babesia and red cell invasion. Curr. Opin. Hematol. 19: 170-175

Lodes M. J., Houghton R. L., Bruinsma E. S., Mohamath R., Reynolds L. D., Benson D. R., Krause P. J., Reed S. G., Persing D. H. (2000) Serological expression cloning of novel immunoreactive antigens of Babesia microti. Infect. Immun. 68: 2783-2790

Montero E., Rodriguez M., Oksov Y., Lobo C. A. (2009) Babesia divergens apical membrane antigen 1 and its interaction with the human red blood cell. Infect. Immun. 77: 4783-4793

Mørch K., Holmaas G., Frolander P. S., Kristoffersen E. K. (2015) Severe human Babesia divergens infection in Norway. Int. J. Infect. Dis. 33: 37-38

Mosqueda J., Olvera-Ramirez A., Aguilar-Tipacamu G., Canto G. J. (2012) Current advances in detection and treatment of babesiosis. Curr. Med. Chem. 19: 1504-1518

Mylonakis E. (2001) When to suspect and how to monitor babesiosis. Am. Fam. Physician. 63: 1969-1974

Okła H., Jasik K. P., Słodki J., Rozwadowska B., Słodki A., Jurzak M., Pierzchała E. (2014) Hepatic tissue changes in rats due to chronic invasion of Babesia microti. Folia Biol. (Kraków). 62: 353-359

Schneider D. A., Yan H., Bastos R. G., Johnson W. C., Gavin P. R., Allen A. J., Barrington G. M., Herrmann-Hoesing L. M., Knowles D. P., Goff W. L. (2011) Dynamics of bovine spleen cell populations during the acute response to Babesia bovis infection: An immunohistological study. Parasite Immunol. 33: $34-44$

Shaio M. F., Lin P. R. (1998) A case study of cytokine profiles in acute human babesiosis. Am. J. Trop. Med. Hyg. 58: 335-337

Skotarczak B. (2007) Babesiosis of human and domestic dog; ethiology, pathogenesis, diagnostics. Wiad. Parazytol. 53: 271-280 (in Polish)

Sonenshine D. E. (1993) Biology of Ticks. Volume 2. Oxford University Press, New York

Teal A. E., Habura A., Ennis J., Keithly J. S., Madison-Antenucci S. (2011) A New Real-Time PCR assay for improved detection of the parasite Babesia microti. J. Clin. Microbiol. 50: 903-908

Terkawi M. A., Cao S., Herbas M. S., Nishimura M., Li Y., Moumouni P. F., Pyarokhil A. H., Kondoh D., Kitamura N., Nishikawa Y., Kato K., Yokoyama N., Zhou J., Suzuki H., Igarashi I., Xuan X. (2015) Macrophages are the determinant of resistance to and outcome of nonlethal Babesia microti infection in mice. Infect. Immun. 83: 8-16 